\title{
The Badger eXploration Loft at Desert RATS 2011
}

\author{
Jordan S. Wachs ${ }^{1,2}$ \\ UW-Madison X-Hab Team ${ }^{3}$ \\ Faculty Advisor: Fred Elder \\ University of Wisconsin-Madison
}

\section{Nomenclature}

$\begin{array}{ll}\text { HDU } & =\text { Habitat Demonstration Unit } \\ \text { DSH } & =\text { Deep Space Habitat } \\ \text { HDU-DSH } & =\text { Habitat Demonstration Unit Deep Space Habitat configuration } \\ \text { D-RATS } & =\text { Desert Research and Technology Studies } \\ \text { X-Hab } & =\text { eXploration Habitat } \\ \text { BXL } & =\text { Badger eXploration Loft } \\ \text { PI } & =\text { Principal Investigator } \\ \text { GeoLab } & =\text { a compact onboard geology laboratory } \\ \text { GMWS } & =\text { General Maintenance Work Station } \\ \text { MOWS } & =\text { Medical Operations Work Station } \\ \text { DMM } & =\text { Dust Mitigation Module }\end{array}$

\begin{abstract}
In recent years, the aim of both NASA as well as the nascent private space industry has become increasingly audacious. By any measure, it is becoming apparent that long duration manned missions to deep space are inevitable; as are the inherent complexities and risks associated with such large scale undertakings. With this in mind, it is critical that engineers and scientists work toward the goal of understanding the difficulties that lay ahead. Analog studies such as the Desert Research and Technology Studies (D-RATS) are one critical resource available for the investigation of the effectiveness of various mission architectures. This paper provides a brief outline of D-RATS and focuses on the role, abilities, and benefits of the integration of the Badger X-Loft with the Habitat Demonstration Unit mission during D-RATS 2011.
\end{abstract}

\footnotetext{
${ }^{1}$ UW-Madison Department of Engineering Physics

${ }^{2}$ UW-Madison Department of Physics

${ }^{3}$ A. Olson Department of Mechanical Engineering, J. Wachs ${ }^{1,2}$, P. Sweeney ${ }^{1}$ Will Yu ${ }^{1}$, A. Arnson, Dept of Design Studies; J. Mason ${ }^{1}$, N.Roth, Granger School of Business; S.Wisser ${ }^{1}$, M. Fritz ${ }^{1}$, S. Marron ${ }^{1}$, M. Lucas ${ }^{1}$, N. Wong ${ }^{1}$

The UW X-Hab team would like to thank the following contributors: Wisconsin Space Grant Consortium, UW Space Science and Engineering Center, UW-Madison Dept. of Engineering Physics, UW-Madiso Dept. of Mechanical Engineering, Ghallager Tent and Awning Company, Air Distribution Concepts
} 


\section{Introduction}

Following the current presidential administration's changes in the prescribed vision for future human exploration of our solar system (Sellers, 2012), the high level objective of Desert RATS 2011 was to test mission architectures for deep space habitation on a manned mission to a near earth asteroid (Gruener, 2012). The annual recurrence of Desert RATS allows teams to rapidly deploy and test new concepts in a safe working environment. Exhibiting a short turn-around time of approximately one year, the "tiger team" approach of the D-RATS

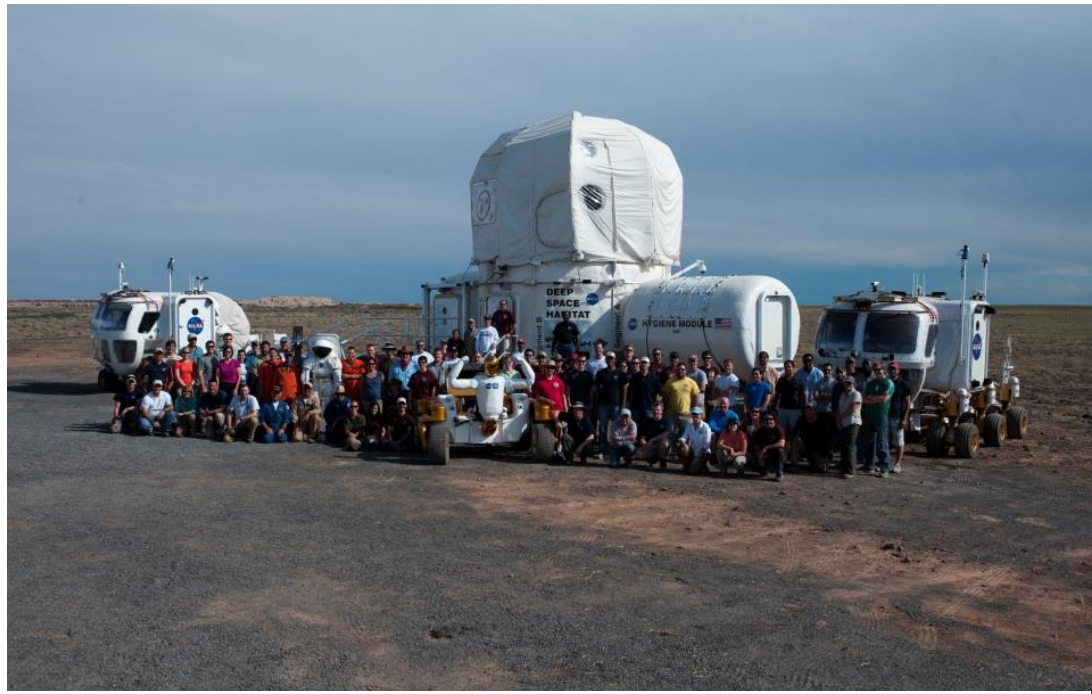

Figure 1: D-RATS team photo with the DHU-DSH, both MMSEVs, and Robonaut.

management allows for faced

paced progression of both technologies and mission goals, promoting flexibility in adaptation to changing high level requirements. Both the simulation's destination and team makeup were changed for 2011, as NASA's mission goals shifted to asteroid exploration. Furthermore, the inaugural X-Hab Academic Challenge provided seed funding for student teams, which brought students directly into the critical path and allowed them to become full-fledged Principal Investigators (PI) for their subsystem under the direction of a faculty advisor (Howe, 2012). Overall, the HDU team, along with the X-Hab team from the University of Wisconsin-Madison, was able to successfully fulfill mission requirements during Desert RATS 2011.

\section{Habitat Demonstration Unit-Deep Space Habitat overview}

The Habitat Demonstration Unit-Deep Space Habitat (HDUDSH) consists of a large number of complex systems spread out through four functional modules. Every module was relatively independent in its operation, with most of the subsystems in each module being confined to that module. A notable exception to this is the avionics package which allows monitoring and interfacing with major systems in all four modules from the same iPad interface.

The HDU itself serves as the primary module, housing most of the mission critical subsystems incorporated within the DSH system. Four large subsystems were given permanent workstations in the HDU; their locations are shown in Figure 2 for reference. The GeoLab, located in the upper right, serves as a

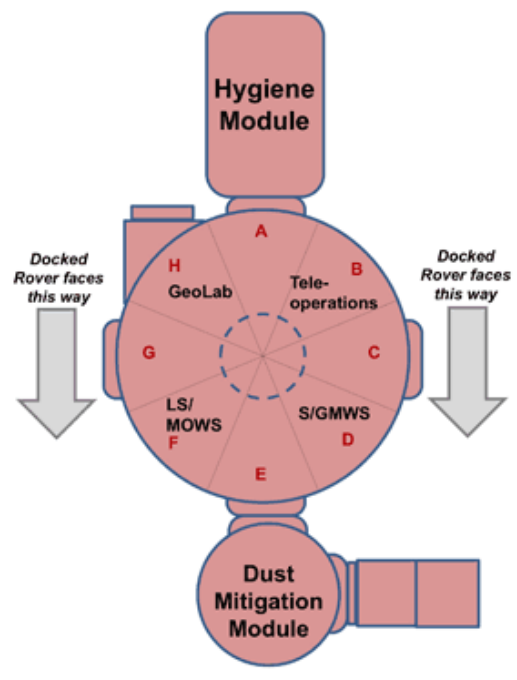

Figure 2: HDU-DSH first level layout 
small but capable laboratory in which scientists can investigate geological samples. Samples are loaded from the exterior of the DSH by either an astronaut or the robotic companion, Robonaut. This configuration allows for investigation of specimens without cross contamination between the environment in the HDU and its exterior. The Tele-operations station allows crew aboard the DSH to communicate with all key players in any given mission scenario: EVA crew, crew aboard the Multi Mission Space Exploration Vehicles (MMSEV), as well as mission control (MCC) in Houston and the on-site mobile MCC. The General Maintenance Work Station, or GMWS provided the ability for crew to perform repair work on mission critical parts, and the MOWS allows crew to conduct medical procedures that may become necessary during the course of the mission. The dashed circle in the center of Figure 2 represents the single person elevator used to get from the lower deck of the DSH to the loft above.

The Dust Mitigation Module (DMM), shown at the bottom of Figure 2 served as a stand-in for what would be an airlock in a live mission. The primary function for the DMM in D-RATS of keeping dust, sand, and dirt from contaminating the interior of the DSH is analogous to keeping fine lunar or asteroid regolith from entering a habitat (Mason, 1992).

A new addition for 2011, the Hygiene Module supplied crew with basic personal hygiene needs. This module incorporated a toilet facility, hand wash station, and full body wash station for extended use.

Situated above the three other modules was the expandable loft, known as the BXL, designed and constructed by the University of Wisconsin-Madison team. The loft served as quarters for the crew while conducting activities not directly associated with mission goals. The lower level of the BXL, the $2^{\text {nd }}$ level of the DSH, was comprised of a communal working area and served many functions. Some of the primary mission systems in the loft include the galley, exercise equipment, general workspace, a secondary Tele-operations station for use during times of high activity on the lower deck, and a crew entertainment station. Above the communal area were situated private quarters for each crew member. This third and highest level of the DSH was a cantilevered partial floor, spanning four feet inward from the outer edge of the BXL at a height sufficient to allow uninhibited crew function beneath. Each crew member was allotted $1 / 4$ of the

$3^{\text {rd }}$ floor for personal use. Air circulation and power were provided in each of the personal crew quarters.

\section{The Badger eXploration Loft}

The requirements supplied to the BXL by the HDU management at NASA were very high level. Such requirements as maximum weight, minimum internal volume, and the high level mission gals were defined, but middle and low level requirements definition was left to the individual teams in order to stimulate creativity in the design process. The benefits seen by NASA management were twofold: Firstly, it was a noted strength of this process that students are often able to think far "outside the box" to come up with ingenuitive ideas because their lack of real world experience means that they aren't aware of what is commonly seen to be impossible.

Secondly, by reaching into the educational system, the HDU team wanted to motivate students to supplement their curricula with real world engineering that would improve the learning experience at each university involved (Howe, 2012). 
One of the greatest assets that the BXL team had was diversity. With students representing various engineering departments, the physics department, the School of Business and the School of Textiles and Interior Design, the team was uniquely positioned to handle the wide range of challenges that came with this large scale design and construction.

Overall, the design itself went through many stages, as the schedule adhered to the NASA review process, including SDR, PDR, and CDR reviews in preparation of the final construction and system testing of the three major subsystems incorporated into the BXL and described below.

Shell. Externally, the most apparent subsystem of the BXL is its shell. This complex soft goods construction was stitched into a single piece before integration with the other subsystems. Incorporating airbeams for structure (shown red in Figure 2), four layers of fabric were suspended with three inch air gaps between each layer to provide insulation against the extreme desert temperatures, and a waterproof exterior fabric for further protection against the elements. This construction proved effective as a lightweight, highly stowable, and reliable solution to the problem of expandable insulation and protection.

Internal Structure. To meet the NASA defined requirement of the ability to withstand 50mph wind loading, and the internally (within

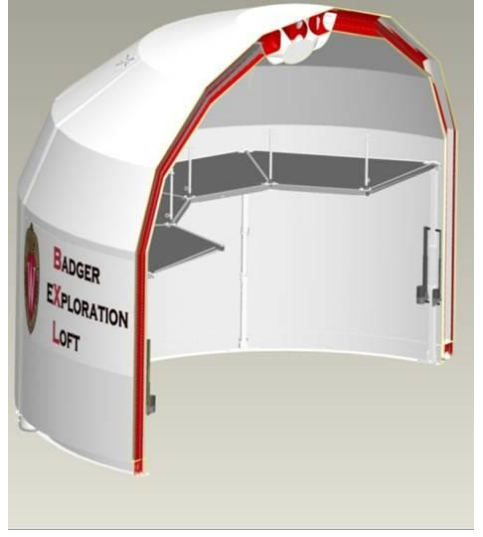

Figure 3: Cutaway rendering showing air gaps and external shell layer the BXL team) defined requirement of a $3^{\text {rd }}$ floor for personal quarters, an internal structure was deemed necessary. This skeletal stucture, comprised of aluminum columns and carbon fiber cantilevered beams and spanners supporting the third floor of the BXL provided ample room for crew to work and live. The internal structure and the shell worked together to fulfill all of the structural and insulation requirements even under conditions far harsher than the design criteria specified, including one storm with winds approaching $70 \mathrm{mph}$.

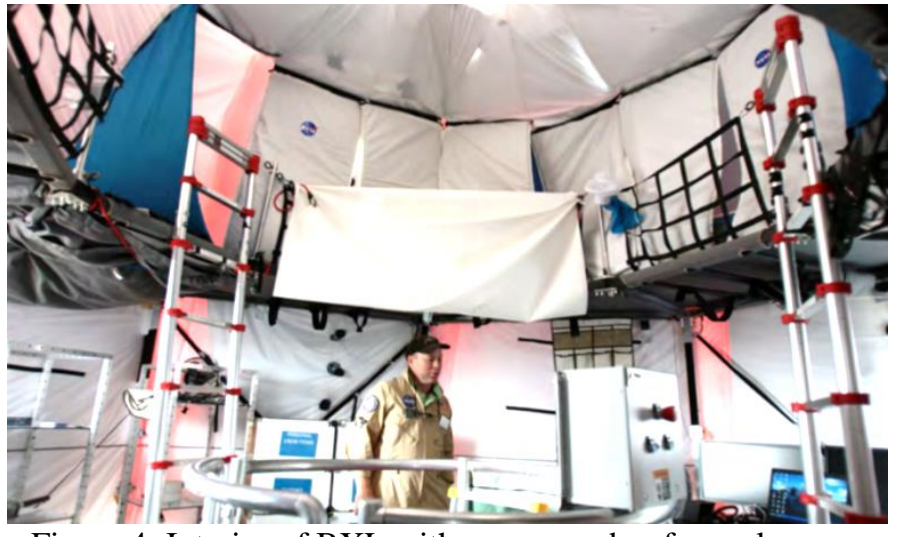

Figure 4: Interior of BXL with crew member for scale

Electrical, Lighting, and Inflation Control (ELIC). The third major subsystem of the BXL, the Electrical, Lighting and Inflation Control subsystem, was very well integrated into the overall habiat, providing dimmable LED lighting to key areas, power outlets where necessary, and a working inflation control system which allowed for testing and integration inflation. Once the BXL had been fully integrated into the DSH, a NASA controlled pressurisation system was integrated and fully networked into the avionics for airbeam pressure monitoring. 


\section{Conclusion}

In conclusion, D-RATS 2011 was a success on many levels. Aside from gathering valuable data pertaining to the future of human rated space exploration, D-RATS validated the approach that the HDU team took to producing innovative hardware while immersing students in the critical path for NASA missions in human spaceflight. The continuation of the eXploration Habitat Academic Innovation Challenge is a clear sign that the Challenge's approach has produced quality work and experience for those involved.

\section{Acknowledgments}

On behalf of the X-Hab team from the University of Wisconsin-Madison, the author would like to thank the WSGC, Fred Best and the University of Wisconsin Space Science and Engineering Center, Randy Illif from Bjorksten|Bit 7, and the members of the Habitat Demonstration Unit team for their contributions to the X-Hab project. Special thanks go to our faculty advisor, Dr. Fred Elder and graduate student advisors Max Salick and Tim Feyereisen for their exceptional guidance through the design and construction processes.

\section{References}

Gruener, J. E. (2012). NASA Desert RATS 2011 Education Pilot Project and Classroom Activities J. E.

Proceedings of the $43^{\text {rd }}$ Lunar and Planetary Science Conference, (p1583). The Woodlands, TX

Howe, A. S. (2012). X-hab Challenge: Students in the Critical Path

American Institute of Aeronautics and Astronautics

Seller, P.J. (2012). A Vision for the Exploration of Mars: Robotic Precursors followed by Humans to Mars Orbit in 2033.

Proceedings of Concepts and Approaches for Mars Exploration from the Lunar and Planetary Institute (p.4140). Houston, TX

Mason, L. W. (1992). Engineering, construction, and operations in space-III: Space '92.

Proceedings of the 3rd International Conference, (pp. 1127-1138). Denver, CO. 